\title{
0863 THE UK BURDEN OF INJURIES STUDY (UKBOI)
}

R A Lyons*, D Kendrick, E L Towner, N Christie, C J Phillips, C Coupland, M Hayes, S Macey, S Brophy, B Carter, L Groom, C Mulvaney, J Sleney, S Smith, J Stewart, P A Evans, I Pallister, F Coffey Correspondence: School of Medicine, Swansea University, Grove Building, SA2 8PP, UK

\subsection{6/ip.2010.029215.863}

Objectives The UKBOI study was designed to quantify important components of the population burden of injury in the UK.

Methods A mixed methods study incorporating a multi-centred prospective cohort of injured individuals; extrapolation of patient reported outcomes to population metrics using routine data from emergency departments (ED), hospital discharge registers and mortality data; calculation of Disability Adjusted Life Years (DALYs) using Global Burden of Diseases methodology and estimated indirect costs; and the calculation of direct medical costs.

Results The study recruited 1517 injured individuals. Estimated numbers (and rates per 100 000) for UK population extrapolations were 750999 (1240) for hospital admissions, 7 982947 (13 339) for emergency department attendances, and 22185 (36.8) for injury related deaths, in the financial year 2005/6. An estimated 1574000 DALYs were lost from injuries occurring in that year, with $80 \%$ due to Years Lived with Disability (YLDs) and 20\% from Years of Life Lost (YLLs). Some $10 \%$ of DALYs lost were due to YLDs occurring within 12 months post injury and $70 \%$ due to lifelong injuries. Direct medical costs were estimated at 482 million for ED visits and 2.15 billion for inpatient stays, with indirect costs of up to 31.5 billion. The qualitative study with 90 participants revealed 
many additional negative consequences for individuals, family and society.

Implications The high population burden and costs associated with injuries in the UK demonstrates the need for sustained policies and interventions in support of prevention. 